\title{
ROLE OF SYMPATHETIC NERVOUS SYSTEM IN OBESITY RELATED HYPERTENSION
}

\author{
Alexandre da Silva, PhD, Jussara doCarmo, PhD, John Dubinion, PhD, and John E. Hall, PhD \\ Department of Physiology and Biophysics and Center of Excellence in Cardiovascular-Renal \\ Research The University of Mississippi Medical Center Jackson, MS
}

\begin{abstract}
Obesity is recognized as a major, worldwide, health problem. Excess weight is a major cause of increased blood pressure in most patients with essential hypertension, and greatly increases the risk for diabetes, cardiovascular diseases, and end stage renal disease. Although the mechanisms by which obesity raises blood pressure are not completely understood, increased renal sodium reabsorption, impaired pressure natriuresis, and volume expansion appear to play important roles. Several potential mechanisms have been suggested to contribute to altered kidney function and hypertension in obesity, including activation of the sympathetic nervous system (SNS) and the renin-angiotensin-aldosterone system (RAAS), and physical compression of the kidneys, especially when visceral obesity is present. Activation of the SNS in obesity may be due, in part, to hyperleptinemia and other factors secreted by adipocytes and the gastrointestinal tract, activation of the central nervous melanocortin pathway, and baroreceptor dysfunction.
\end{abstract}

\section{INTRODUCTION}

In the past two decades we have witnessed a rapid increase in the prevalence of obesity in developing as well as industrialized countries. Approximately 34\% of U.S. adults aged 20 and over are obese with body mass index (BMI) greater than 30 and almost $2 / 3$ of the adult population is overweight. [1**] Even more alarming is the fact that the numbers of overweight and obese children and adolescents have tripled in the last three decades [1**]; obese children are likely to stay obese into adulthood and develop cardiovascular diseases and diabetes at a young age.

Being overweight or obese increases the risk for cardiovascular disease through multiple mechanisms, including diabetes, dyslipidemia, atherosclerosis, renal disease, and hypertension [2,3]. Although these disorders often occur together and have been termed the "metabolic syndrome", they are almost always initiated by obesity. For instance, risk estimates from population studies suggest that excess weight gain may contribute to as much as $65 \%$ to $75 \%$ of the risk for essential hypertension and up to $80 \%$ to $90 \%$ of the risk for diabetes and other metabolic disorders [2]. Additional considerations that are sometimes overlooked but may cause underestimation of the impact of obesity on cardiovascular risk include the duration of obesity and body fat distribution. For example, the risk for developing diabetes increases progressively with increasing duration of obesity [4]. Likewise, visceral obesity confers a greater risk for the development of hypertension and cardiovascular disease compared to subcutaneous or lower body obesity [5,6**].

Please address all correspondence to: Dr. Alexandre A. da Silva Department of Physiology and Biophysics University of Mississippi Medical Center 2500 North State St. Jackson, MS 39216-4505 Phone: 601-984-1826 Fax: 601-984-1817 asilva@physiology.umsmed.edu. 
Although hypertension is much more prevalent in obese than in lean populations [7,8], not all obese individuals are hypertensive as assessed by standard clinical criteria. One potential explanation for this apparent variable effect of obesity on hypertension is that weight gain causes a shift in the Gaussian distribution of blood pressure towards higher values such that an obese individual who may not be classified as being hypertensive would have a lower blood pressure at a lower body weight [5]. In fact, weight loss is associated with a reduction in blood pressure even in normotensive obese subjects [9]. Another possibility is the inter-individual variability in body fat distribution which, as mentioned previously, could confer a stronger or weaker association between body weight and increased blood pressure depending on where the adipose tissue is located.

The mechanisms by which excess weight gain elevates arterial pressure are incompletely understood. Obesity related hypertension is associated with sodium retention and impaired renal pressure natriuresis (i.e. rightward shift in the pressure-natriuresis curve toward hypertensive levels) $[10,11]$. How excess weight gain leads to increased sodium reabsorption is unclear but appears to be caused, at least in part, by activation of the renin-angiotensinaldosterone system (RAAS), physical compression of the kidneys, and increased sympathetic nervous system (SNS) activity. In this brief review we will focus mainly on the role of increased SNS activity in contributing to the development of hypertension in obesity.

\section{Sympathetic Nervous System Activation in Obesity Hypertension}

Most recent studies indicate that excess weight gain, especially when associated with increased visceral adiposity, is associated with increased SNS activation and that this increase in SNS activity contributes to the development of hypertension in obese humans as well as in animal models of diet-induced obesity $\left[6^{* *}, 12\right]$. Even modest weight gain in nonobese subjects is associated with increased SNS activity [6**].

Pharmacological antagonism of adrenergic receptors causes greater reductions in blood pressure in obese compared to lean hypertensive subjects [13], and renal sympathetic denervation markedly attenuates the sodium and water retention and the rise in arterial pressure in a high fat diet model of obesity in dogs [14]. Collectively, these results provide strong evidence for SNS activation in obesity and that increased renal SNS activity is a primary mechanism for elevated blood pressure in obesity rather than SNS activation in other tissues with associated peripheral vasoconstriction. In fact, obesity may not lead to generalized SNS activation but to a selective and differentiated increase in sympathetic activity. For example, in obese humans SNS activity in skeletal muscle and the kidneys is elevated whereas cardiac sympathetic activity is barely elevated or may even be reduced due to baroreflex inhibition [15]; the increased heart rate associated with obesity is mediated mainly by reduced parasympathetic tone to the heart, $\left[3,6^{* *}\right]$.

Visceral obesity elicits greater SNS activation than subcutaneous obesity [16]. The mechanisms responsible for the link between visceral obesity and SNS activation have not been widely studied, and in most human studies muscle SNS activity has been measured rather than renal SNS activity, the primary pathway by which the SNS causes chronic hypertension [3,14]. Because there is considerable heterogeneity in the control of autonomic outflow to different organs, measurements of muscle SNS activity may not necessarily reflect renal SNS activity. A comprehensive analysis of the factors that influence the relationships among body fat distribution, differential regulation of SNS activity, and blood pressure has, to our knowledge, not been conducted. 


\section{Mechanisms of Sympathetic Activation in Obesity}

Although the rise in arterial pressure caused by excess weight gain is mediated, at least in part, by increased SNS activity, the factors that link obesity with renal sympathetic activation are unclear. Several potential factors have been proposed to contribute to increased SNS activity in obesity, including hyperleptinemia, activation of the central pro-opiomelanocortin/ melanocortin-4 receptor (POMC/MC4R), hyperinsulinemia/insulin resistance, hypoadiponectinemia, hypoghrelinemia, increased angiotensin II levels, and baroreceptor dysfunction (Figure 1).

Hyperleptinemia—Leptin is secreted by adipocytes in proportion to fat mass and acts on multiple centers in the central nervous system (CNS), especially within the hypothalamus, to decrease appetite and increase energy expenditure [17]. Despite evidence that obesity is associated with reduced responsiveness to the anorexic effects of leptin, the ability of leptin to raise renal sympathetic activity appears to be intact in obesity [refer to ref. 18 for detailed description of selective leptin resistance].

Although its stimulatory effects on SNS activity are clearly documented, leptin appears to have little effect on blood pressure when acutely injected into experimental animals [19]. The absence of acute pressor responses may be due, in part, to counteracting vasodilatory effects of nitric oxide (NO) stimulated by leptin [20]. Also, leptin-mediated increases in sympathetic activity may not be great enough to cause marked peripheral vasoconstriction and acutely raise arterial pressure.

In contrast to the absence of acute hemodynamic effects, chronic infusion of leptin in lean rodents to achieve plasma levels that mimic those observed in obesity causes slow and gradual elevations in arterial pressure that can be abolished by chronic adrenergic blockade [21]. Moreover, the chronic effects of leptin to raise blood pressure and heart rate were markedly augmented in rats receiving the inhibitor of nitric oxide synthesis L-NAME [22]. These observations suggest that impairment of endothelial function and NO production, as may occur in obese subjects, amplifies the chronic hypertensive effects of leptin.

Evidence for a role of leptin in long-term regulation of SNS activity and arterial pressure also comes from studies showing that leptin deficiency is associated with early-onset morbid obesity and metabolic syndrome without SNS activation or hypertension [23]. In fact, these individuals show impaired sympathetic activity, postural hypotension, and attenuated renin-angiotensin responses to upright posture [23]. Hypertension does not develop even in the presence of severe insulin resistance and hyperinsulinemia. Similar observations in leptin-deficient mice or in rodents with leptin receptor mutations suggest that hyperleptinemia may be an important link between obesity, SNS activation and hypertension.

Activation of the CNS Melanocortin System-The stimulatory effect of leptin on SNS activity appears to depend on interactions with other CNS factors, specifically the proopiomelanocortin system (POMC) system. Acute blockade of the melanocortin 3 and 4 receptors $(\mathrm{MC} 3 / 4 \mathrm{R})$ using a pharmacological antagonist infused directly into the cerebral ventricles completely abolished the leptin-induced increase in renal SNS activity [24], while chronic blockade of the MC3/4-R in rats caused rapid weight gain and marked bradycardia and abolished the rise in blood pressure usually observed during chronic hyperleptinemia [25]. In another study, Tallam et al. showed that the melanocortin-4 receptor (MC4R) is the key melanocortin receptor involved in regulation of appetite, metabolic and cardiovascular function since MC4R deficient mice are obese but do not have hypertension despite hyperphagia, hyperinsulinemia and hyperleptinemia [26]. In addition, MC4R deficient mice are completely unresponsive to the dietary, metabolic and cardiovascular effects of chronic leptin infusion 
[27*]. Thus, an intact POMC-MC4R pathway is required for excess visceral adiposity and hyperleptinemia to increase SNS activity and arterial pressure in rodents.

A recent study also indicates that the prevalence of hypertension is markedly lower in MC4R deficient humans compared to obese control subjects, despite the presence of severe obesity and its associated metabolic disorders [28**]. Even after exclusion of subjects taking antihypertensive medications, blood pressure and heart rate levels were significantly lower in MC4R-deficient subjects than in control subjects. Moreover, subcutaneous administration of a MC4R agonist for 7 days caused significant increases in blood pressure, similar to those observed in rodents. Thus, in humans and rodents, chronic activation of the MC4R raises blood pressure and the presence of a functional POMC-MC4R system appears to be necessary for obesity to increase SNS activity and arterial pressure.

Support for a role of the CNS melanocortin system in the development of spontaneous hypertension comes from a recent study showing that endogenous baseline activation of the MC3/4R contributes to hypertension in spontaneously hypertensive rats (SHR), a rodent model of hypertension associated with increased SNS activity [29*]. Chronic antagonism of MC3/4R in SHR caused a marked reduction in blood pressure similar to that achieved with adrenergic blockade [29*]. Although the SHR is not considered to be obese since its total body weight is usually lower than those of Wistar-Kyoto or Sprague-Dawley control rats, this model of hypertension is associated with increased visceral adiposity, SNS activation, insulin resistance, increased leptin, and other metabolic abnormalities that are often associated with obesity. Even if the SNS activation in SHR is not directly linked to visceral adiposity, it is clear that activation of the MC3/4R contributes to increased SNS activity and hypertension in this model. The role of MC3/4R activation in non-obese genetically hypertensive humans has not been assessed.

Hypoadiponectinemia - Like leptin, adiponectin is secreted by adipocytes and may play a role in long-term regulation of arterial pressure [30**]. Adiponectin circulates in several multimeric complex forms that appear to have distinct functions, and high molecular weight adiponectin appears to mediate the beneficial metabolic and cardiovascular effects of adiponectin [31]. In contrast to leptin, high molecular weight adiponectin levels are reduced in obesity and adiponectin deficiency appears to have detrimental effects on metabolism and cardiovascular function [30**].

Although adiponectin-deficient mice are not hypertensive when fed a normal salt intake, they are salt-sensitive and have higher systolic blood pressure when exposed to high salt intake. Increasing adiponectin levels using an adenoviral construct attenuated salt-induced increases in blood pressure in adiponectin-deficient mice. Adiponectin supplementation also reduced systolic blood pressure in obese KKay mice [32,33]. Although the mechanisms by which hypoadiponectinemia alters blood pressure regulation are not completely understood, activation of the RAAS, endothelial dysfunction and increased SNS activity may be involved [30**]. Evidence for a role of adiponectin in regulating SNS activity is limited to acute studies showing that CNS injections of adiponectin reduce renal SNS activity and blood pressure [34]. Whether chronic increases in CNS levels of adiponectin can cause sustained reductions in renal SNS activity and blood pressure is unknown and remains an important area for further investigation.

Hypoghrelinemia-Ghrelin, a peptide produced by the stomach, rises during fasting and appears to plays a key role in meal initiation. In addition to triggering hunger, acute ghrelin infusion into the CNS of rodents reduces SNS activity and counteracts the stimulatory effects of leptin on the POMC-MC4R pathway [35-37]. 
Ghrelin levels are reduced in obesity, perhaps as a compensation for excess energy intake due to more frequent and larger meals. This has led to the hypothesis that reduced circulating ghrelin may contribute to SNS activation and the development of hypertension in obese subjects. In addition to potentially lowering SNS activity, ghrelin may also contribute to the regulation of arterial pressure by increasing NO production via activation of endothelial nitric oxide synthase [38]. However, to our knowledge, no long-term studies have investigated the effects of ghrelin on control of SNS activity and arterial pressure, or have tested whether ghrelin deficiency in the absence of weight loss is associated with alterations in cardiovascular function, including hypertension.

Hyperinsulinemia-Insulin resistance and hyperinsulinemia have been hypothesized to play an important role in SNS activation and hypertension in obesity. However, key observations in experimental animal models as well as in humans do not support a role for hyperinsulinemia in the etiology of obesity hypertension. For instance, blood pressure does not rise when insulin is chronically infused in dogs or in humans treated with insulin [39]. Also, humans with insulinoma, in whom fasting insulin levels are 4- to 5-fold higher than normal, do not exhibit increased SNS activity or elevated arterial pressure [5]. Although CNS injection of large amounts of insulin in rodents may raise renal SNS activity acutely, this effect may not be longlasting since chronic CNS infusion of insulin at varying doses failed to alter arterial pressure or heart rate [40]. These results do not exclude the possibility that insulin resistance may lead to endothelial dysfunction and other vascular abnormalities that in turn could contribute to the obesity related renal disease and hypertension. However, as discussed previously, several rodent models as well as humans with severe insulin resistance and hyperinsulinemia but without a functional leptin-CNS melanocortin system do not appear to have SNS activation or hypertension, at least prior to the development of renal injury $[23,26]$.

Increased Angiotensin II levels-Activation of the RAAS appears to play an important role in the etiology of obesity related hypertension by direct effects on the kidney to promote increased sodium reabsorption as well as by stimulation of aldosterone secretion [41-44]. Increased angiotensin II levels in obesity have also been suggested to cause SNS activation. Acute angiotensin II infusion in normotensive humans raises muscle SNS activity and blockade of the RAAS reduces muscle SNS activity. However, Lohmeier et al. showed that chronic angiotensin II infusion in dogs reduced rather than increased renal norepinephrine spillover [45] and that chronic baroreflex activation to suppress SNS activity caused a more pronounced fall in blood pressure in control dogs than in dogs infused with angiotensin II [46], suggesting that the angiotensin II-hypertension is associated with reduced rather than increased SNS activity. It is possible that in the presence of baroreflex dysfunction, as occurs in obesity, the buffering effects of baroreflex activation may be reduced, leading to increased rather than reduced SNS activity when circulating angiotensin II levels are elevated. In dogs with cardiopulmonary and sinoaortic baroreceptor denervation angiotensin II infusion increases SNS activity [47]. Although angiotensin II contributes importantly to obesity-induced hypertension, additional studies are needed to determine if this is mediated by SNS activation, by direct effects on the kidney, or by stimulation of aldosterone secretion.

Baroreflex dysfunction-Baroreflex control of SNS activity plays a key role in acute buffering of fluctuations of blood pressure, but its importance in the long-term control of blood pressure is less clear. In obese humans, baroreflex function is impaired and the degree of impairment appears to be associated with body fat distribution; subjects with abdominal obesity have greater reduction in baroreflex sensitivity than those with peripheral obesity [16]. Chronic activation of the baroreflex with electrical stimulation also markedly attenuated the rise in blood pressure in obesity-induced hypertension in dogs [48]. These results are consistent with a role for baroreflex dysfunction in contributing to obesity related hypertension. However, the 
rapid adaption of the baroreflex during sustained changes in arterial pressure and the limited relevance of acute assessment of baroreflex sensitivity to understanding its role in long-term blood pressure regulation make it difficult to assess the importance of baroreflex dysfunction in obesity hypertension in humans.

\section{Treatment of Obesity Related Hypertension}

Due to the complex etiology of obesity-induced hypertension and the paucity of relevant clinical trial data there are no specific guidelines for pharmacological treatment of obese patients, and physicians generally rely on their personal judgment for the most appropriate treatment. Based on the clear evidence that activation of the RAAS and SNS play a key role in mediating increased blood pressure during excess weight gain, pharmacological blockade of these systems are logical choices. [For a comprehensive review on the potential benefits and pitfalls of targeting the SNS for the treatment of obesity related hypertension please refer to reference 49].

Non-pharmacological therapies including weight loss and increased physical activity have been shown to be effective in lowering arterial pressure, and even modest reduction in body weight significantly reduces both systolic and diastolic blood pressures [5]. However, it is very difficult to maintain a lower body mass after having been obese, and most patients regain weight after periods of intense to moderate weight loss [50]. Thus, the best way to reduce the burden of obesity hypertension is prevention of obesity rather than treatment of obesity and related hypertension. However, until effective strategies for preventing and treating obesity are developed, the cardiovascular and renal consequences of obesity must be aggressively treated.

\section{CONCLUSIONS}

Although excess weight gain is recognized as one of the most important risk factors for the development of hypertension, the mechanisms by which obesity raises arterial pressure are not fully understood. Endothelial dysfunction, physical compression of the kidneys and activation of the RAAS have all been proposed to be important. Evidence also suggests that excess weight gain leads to increased SNS activity, that renal denervation markedly attenuates the rise in blood pressure in dietary-induced models of obesity, and that adrenergic receptor blockade reduces blood pressure to a greater extent in obese compared to lean subjects.

The mechanisms leading to SNS activation in obesity appear to involve elevations in circulating leptin levels and activation of the CNS POMC-MC4R pathway since experimental models of obesity or obese humans with deficiency of leptin or of POMC-MC4R usually do not have increased SNS activation or hypertension. Other factors, such as reduced NO formation, increased angiotensin II levels, baroreflex dysfunction, and reduction in adiponectin and ghrelin levels may also contribute to SNS activation in obesity. It is likely that these factors act in concert to potentiate their individual effects on SNS activity and blood pressure.

\section{Acknowledgments}

The authors' research was supported by a grant from the National Heart, Lung and Blood Institute (P01 HL 51971) and by an American Heart Association Scientist Development Grant to Alexandre A. da Silva (0635600N).

\section{REFERENCE LIST}

**1. Department of Health and Human Services - Center for disease Control and PreventionObesity and overweight trends in the U.S2008Available at: http://www.cdc.gov/nccdphp/dnpa/obesity/trend/index.htm This website maintained by the Department of Health and Human Services contains resourceful and updated information on obesity 
and overweight trends in U.S. adults and children, and also provides many other statistics related to the prevalence of obesity in the United States.

2. Wilson PWF, D'Agostino RB, Sullivan L, et al. Overweight and obesity as determinants of cardiovascular risk - the Framingham experience. Arch Intern Med 2002;162:1867-1872. [PubMed: 12196085]

3. Hall JE, Crook ED, Jones DW, et al. Mechanisms of obesity-associated cardiovascular and renal disease. Am J Med Sci 2002;324(3):127-137. [PubMed: 12240710]

4. Wannamethee SG, Shaper GA. Diabetes Care 1999;22:1266-1272. [PubMed: 10480769]

5. Davy KP, Hall JE. Obesity and hypertension: two epidemics or one? Am J Physiol Regul Integr comp Physiol 2004;286:R803-R813. [PubMed: 15068965]

**6. Davy KP, Orr JS. Sympathetic nervous system behavior in human obesity. Neurosci Biobehav Rev 2009;33:116-124. [PubMed: 18602694] Provides a comprehensive review of differential control of sympathetic activity in human obesity with emphasis on the effects of body fat distribution (visceral versus peripheral obesity).

7. Alexander, J.; Dustan, HP.; Sims, EAH., et al. Report of the Hypertension Task force. Washington, DC: 1979. p. 61-77.US Government Printing Office, US Department of Health, Education, and Welfare Publication No. 70-1631 (NIH)

8. Jones DW, Kim JS, Andrew ME, et al. Body mass index and blood pressures in Korean men and women: The Korean National Blood Pressure Survey. J Hypertens 1994;12:1433-1437. [PubMed: 7706705]

9. Neter JE, Stam BE, Kok FL, et al. Influence of weight reduction on blood pressure: a meta-analysis of randomized controlled trials. Hypertension 2003;42:878-884. [PubMed: 12975389]

10. Guyton AC. The surprising kidney-fluid mechanism for pressure control: Its infinite gain. Hypertension 1990;16:425-730.

11. Hall JE. The kidney, hypertension, and obesity. Hypertension 2003;41:625-633. [PubMed: 12623970]

12. Hall JE, Brands MW, Hildebrandt DA, et al. Role of sympathetic nervous system and neuropeptides in obesity hypertension. Braz J Med Biol Res 2000;33:605-618. [PubMed: 10829088]

13. Wofford MR, Anderson DC, Brown CA, et al. Antihypertensive effect of alpha and beta adrenergic blockade in obese and lean hypertensive subjects. Am J Hypertens 2001;14:694-698. [PubMed: 11465655]

14. Kassab S, Kato T, Wilkins C, et al. Renal denervation attenuates the sodium retention and hypertension associated with obesity. Hypertension 1995;25:893-897. [PubMed: 7721450]

15. Straznicky NE, Eikelis N, Lambert EA, et al. Mediators of sympathetic activation in metabolic syndrome obesity. Curr Hypertens Rep 2008;10:440-447. [PubMed: 18959829]

16. Grassi G, Dell'Oro R, Facchini A, et al. Effect of central and peripheral obesity body fat distribution on sympathetic and baroreflex function in obese normotensives. J Hypertens 2004;22:2363-2369. [PubMed: 15614031]

17. Schwartz MW, Woods SC, Porte D Jr. et al. Central nervous system control of food intake. Nature 2000;404:661-671. [PubMed: 10766253]

18. Correia ML, Rahmouni K. Role of leptin in the cardiovascular and endocrine complications of metabolic syndrome. Diabetes Obes Metab 2006;8:603-610. [PubMed: 17026484]

19. Rahmouni K, Haynes WG, Morgan DA, et al. Intracellular mechanisms involved in leptin regulation of sympathetic outflow. Hypertension 2003;41:763-767. [PubMed: 12623993]

20. Kimura K, Tsuda K, Baba A, et al. Involvement of nitric oxide in endothelium-dependent arterial relaxation by leptin. Biochem Biophys Res Commun 2000;273:745-749. [PubMed: 10873674]

21. Carlyle M, Jones OB, Kuo JJ, et al. Chronic cardiovascular and renal actions of leptin-role of adrenergic activity. Hypertension 2002;39:496-501. [PubMed: 11882597]

22. Kuo JJ, Jones OB, Hall JE. Inhibition of NO synthesis enhances chronic cardiovascular and renal actions of leptin. Hypertension 2001;37:670-676. [PubMed: 11230354]

23. Ozata M, Ozdemir IC, Licinio J. Human leptin deficiency caused by a missense mutation: multiple endocrine defects, decreased sympathetic tone, and immune system dysfunction indicate new targets for leptin action, greater central than peripheral resistance to the effects of leptin, and spontaneous 
correction of leptin-mediated defects. J Clin Endocrinol Metab 1999;10:3686-3695. [PubMed: $10523015]$

24. Haynes WG, Morgan DA, Djalali A, et al. Interactions between the melanocortin system and leptin in control of sympathetic nerve traffic. Hypertension 1999;33:542-547. [PubMed: 9931162]

25. da Silva AA, Kuo JJ, Hall JE. Role of hypothalamic melanocortin 3/4-receptors in mediating chronic cardiovascular, renal, and metabolic actions of leptin. Hypertension 2004;43:1312-1317. [PubMed: 15123576]

26. Tallam LS, Stec DE, Willis MA, et al. Melanocortin-4 receptor-deficient mice are not hypertensive or salt-sensitive despite obesity, hyperinsulinemia, and hyperleptinemia. Hypertension 2005;46:326332. [PubMed: 16027245]

*27. Tallam LS, da Silva AA, Hall JE. Melanocortin-4 receptor mediates chronic cardiovascular and metabolic actions of leptin. Hypertension 2006;48:58-64. [PubMed: 16754792] This study shows that melanocortin-4 receptor deficient mice are completely unresponsive to the chronic effects of leptin on appetite, metabolic and cardiovascular functions.

**28. Greenfield JR, Miller JW, Keogh JM, et al. Modulation of blood pressure by central melanocortinergic pathways. N Engl J Med 2009;360:44-52. [PubMed: 19092146] This study is the first to report blood pressure regulation in humans with melanocortin-4 receptor deficiency, and shows that obese humans who lack melanocortin-4 receptors have reduced diastolic and systolic blood pressures compared to obese controls.

*29. da Silva AA, do Carmo JM, Kanyicska B, et al. Endogenous melanocortin system activity contributes to the elevated arterial pressure in spontaneously hypertensive rat. Hypertension 2008;51:884-890. [PubMed: 18285617] This study examined the importance of the endogenous baseline activity of the CNS melanocortin system in maintaining the hypertension in the SHR, and suggests that higher endogenous activation of the CNS melanocortin pathway may also be an important component in other forms of hypertension associated with increased SNS activity.

**30. Wang ZV, Scherer PE. Adiponectin, Cardiovascular Function, and Hypertension. Hypertension 2008;51:8-14. [PubMed: 17998473] This paper reviews the potential actions of adiponectin and hypoadiponectinemia on cardiovascular function, including effects on endothelial function, activation of the renin-angiotensin-aldosterone system, and regulation of sympathetic nervous system activity.

31. Pajvani UB, Hawkins M, Combs TP, et al. Complex distribution, not absolute amount of adiponectin, correlates with thiazolidinedione-mediated improvement in insulin sensitivity. J Biol Chem 2004;279:12152-12162. [PubMed: 14699128]

32. Ohashi K, Kihara S, Ouchi N, et al. Adiponectin replenishment ameliorates obesity-related hypertension. Hypertension 2006;47:1108-1116. [PubMed: 16651465]

33. Ouchi N, Ohishi M, Kihara S, et al. Association of hypoadiponectinemia with impaired vasoreactivity. Hypertension 2003;42:231-234. [PubMed: 12860835]

34. Tanida M, Shen J, Horii Y, et al. Effects of adiponectin on the renal sympathetic nerve activity and blood pressure in rats. Exp Biol Med 2007;232:390-397.

35. Lin Y, Matsumura K, Fukuhara M, et al. Ghrelin acts at the nucleus of the solitary tract to decrease arterial pressure in rats. Hypertension 2004;43:977-982. [PubMed: 14993197]

36. Matsumura K, Tsuchihashi T, Fujii K, et al. Central ghrelin regulates sympathetic activity in conscious rabbits. Hypertension 2002;40:694-699. [PubMed: 12411464]

37. Gil-Campos M, Aguilera CM, Cañete R, et al. Ghrelin: a hormone regulating food intake and energy homeostasis. Br J Nutr 2006;96:201-226. [PubMed: 16923214]

38. Xu X, Jhun BS, Ha CH, et al. Molecular mechanisms of ghrelin-mediated endothelial nitric oxide synthase activation. Endocrinology 2008;149:4183-4192. [PubMed: 18450953]

39. Hall JE, Summers RL, Brands MW, Keen H, Alonso-Galicia M. Resistance to metabolic actions of insulin and its role in hypertension. Am J Hypertens 1994;7:772-88. [PubMed: 7986471]

40. Liu J, da Silva AA, Tallam LS, et al. Chronic central nervous system hyperinsulinemia and regulation of arterial pressure and food intake. J Hypertens 2006;24:1391-1395. [PubMed: 16794489]

41. Hall JE, Jones DW, Kuo JJ, et al. Impact of the obesity epidemic on hypertension and renal disease. Curr Hypertens Rep 2003;5:386-392. [PubMed: 12948431] 
42. Umemura S, Nyui N, Tamura K, et al. Plasma angiotensinogen concentrations in obese patients. Am J Hypertens 1997;10:629-633. [PubMed: 9194508]

43. Reisin E, Weir MR, Falkner B, et al. Lisinopril versus hydrochlorothiazide in obese hypertensive patients: a multicenter placebo-controlled trial. Treatment in Obese Patients With Hypertension (TROPHY) Study Group. Hypertension 1997;30:140-145. [PubMed: 9231834]

44. de Paula RB, da Silva AA, Hall JE. Aldosterone antagonism attenuates obesity-induced hypertension and glomerular hyperfiltration. Hypertension 2004;43:41-47. [PubMed: 14638627]

45. Carroll RG, Lohmeier TE, Brown AJ. Chronic angiotensin II infusion decreases renal norepinephrine overflow in the conscious dog. Hypertension 1984;6:675-681. [PubMed: 6500673]

46. Lohmeier TE, Dwyer TM, Hildebrandt DA, et al. Influence of prolonged baroreflex activation on arterial pressure in angiotensin hypertension. Hypertension 2005;46:1194-1200. [PubMed: 16216987]

47. Lohmeier TE, Lohmeier JR, Haque A, et al. Baroreflexes prevent neurally induced sodium retention in angiotensin hypertension. Am J Physiol Regul Integr Comp Physiol 2000;279:R1437-R1448. [PubMed: 11004014]

48. Lohmeier TE, Dwyer TM, Irwin ED, et al. Prolonged activation of the baroreflex abolishes obesityinduced hypertension. Hypertension 2007;49:1307-1314. [PubMed: 17438305]

$* * 49$. Biaggioni I. Should we target the sympathetic nervous system in the treatment of obesity-associated hypertension. Hypertension 2008;51:168-171. [PubMed: 18086947] This is a short comprehensive review on the pros and cons of antihypertensive therapy targeting the sympathetic nervous system in obese hypertensive patients.

50. Svetkey LP, Stevens VJ, Brantley PJ, et al. Comparison of strategies for sustaining weight loss: the weight loss maintenance randomized controlled trial. JAMA 2008;299:1139-1148. [PubMed: 18334689] 


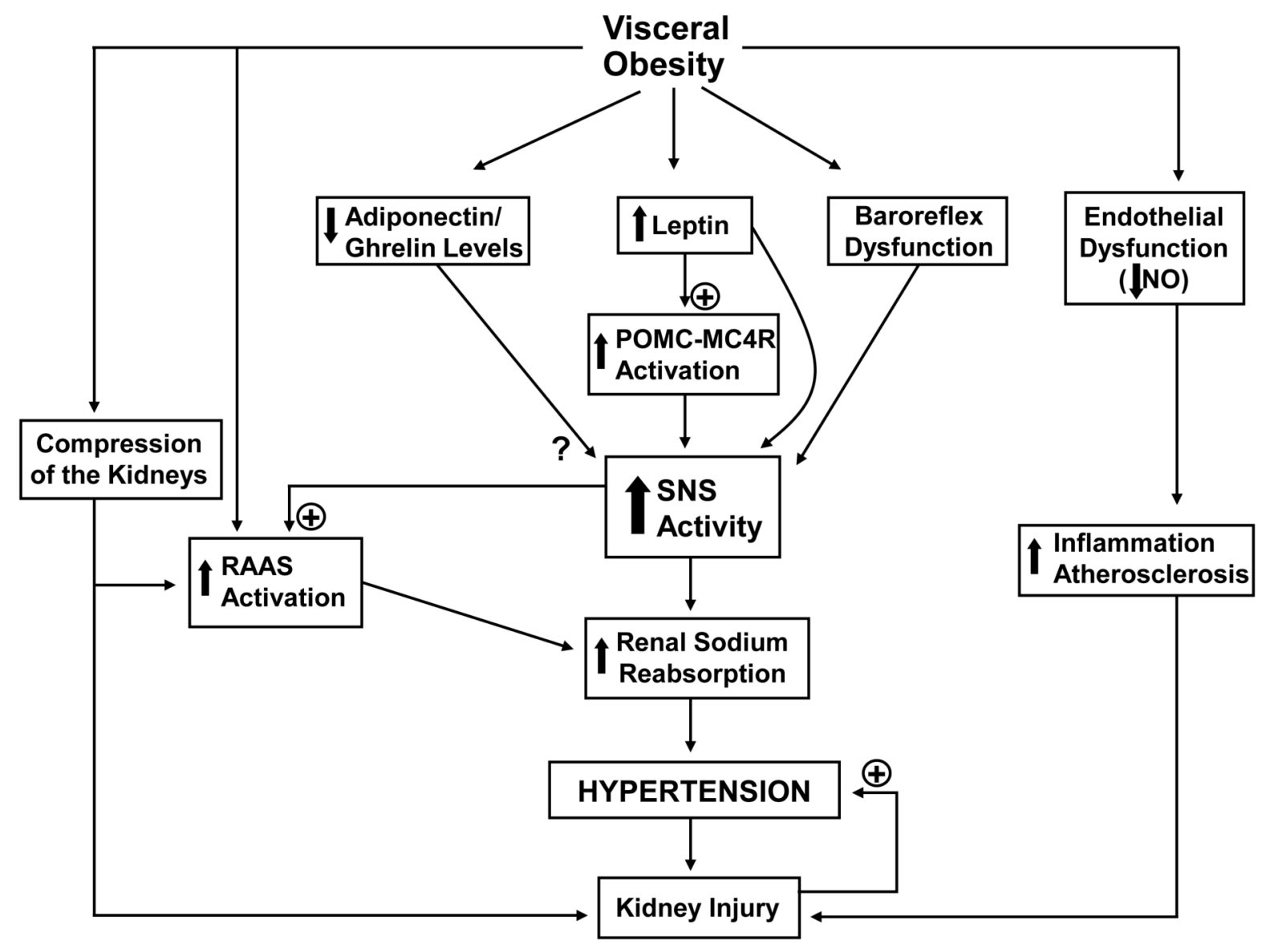

Figure 1.

Potential mechanisms of obesity related hypertension and increased SNS activity. RAAS indicates renin-angiotensin-aldosterone system; SNS indicates sympathetic nervous system; NO indicates nitric oxide; POMC-MC4R indicates pro-opiomelanocortin-melanocortin 4 receptor. 